\title{
CHARAKTERISIERUNG VON ORGANOKIESELSÄUREPOLYMEREN DURCH THERMOANALYTISCHE UNTERSUCHUNGEN
}

\author{
P. Kölsch ${ }^{* 1}$, I. Pitsch ${ }^{1}$, D. Schultze ${ }^{2}$, D. Heidemann ${ }^{3}$ und \\ D. Hoebbel ${ }^{4}$ \\ ${ }^{1}$ Institut für Angewandte Chemie, D-12489 Berlin \\ ${ }^{2}$ Bundesanstalt für Materialforschung und -Prüfung, D-12489 Berlin \\ ${ }^{3}$ Institut für Baustoffkonstruktion und Festigkeit, Technische Universität, D-12489 Berlin, \\ ${ }^{4}$ Institut für Neue Materialien, D-66123 Saarbrücken, F.R.G.
}

(Eingegangen am 15. Juli 1993)

\begin{abstract}
Four organosilicate polymers synthesized by addition of vinyl- and $\mathrm{H}$-substituted double four-ring silicic acid derivatives were characterized using DSC and simultaneous TG-DTA measurements. Thermooxidative decomposition proceeds in several steps: Oxidation of ( $\mathrm{Si}-\mathrm{H})$ groups, oxidation of $\left(\mathrm{Si}-\mathrm{CH}_{2}-\mathrm{CH}_{2}-\mathrm{Si}\right)$ bridges, and oxidation of $\left(\mathrm{Si}-\mathrm{CH}_{3}\right)$ groups, with formation of new ( $\mathrm{Si}-\mathrm{O}-\mathrm{Si}$ ) bonds. ${ }^{29} \mathrm{Si}-\mathrm{NMR}$-spectroscopic measurements confirm this interpretation. Thermoanalytical methods of investigation proved useful to characterize these organosilicate polymers.
\end{abstract}

Keywords: double four-ring silicate units, DSC, organosilicic acid, polymer, ${ }^{29} \mathrm{Si}-\mathrm{NMR}$, TGDTA, thermal analysis

\section{Einleitung}

Die im folgenden untersuchten Organokieselsäurepolymere gehören zu einer neuen Stoffgruppe, die durch dreidimensionale Vernetzung von Doppelvierring- (D4R-) Kieselsäureeinheiten $\left(\mathrm{Si}_{8} \mathrm{O}_{20}\right)$ gebildet werden. An vier ausgewählten Organokieselsäurepolymeren, die sich wesentlich in der Struktur und Länge der Brücken zwischen den D4R-Einheiten sowie im Umsatzgrad der

\footnotetext{
- Korrespondenzanschrift: Institut für Angewandte Chemie, Rudower Chaussee 5, D-12489 Berlin, Bundesrepublik Deutschland
} 
Polyaddition unterscheiden, wurden thermoanalytische Untersuchungen vorgenommen, über die berichtet werden soll.

\section{Experimenteller Teil}

Herstellung, Aufbau und adsorptive Charakterisierung der Organokieselsäurepolymere wurden in den Arbeiten [1-4] ausführlich beschrieben. Im folgenden werden die Ergebnisse bezüglich der Synthese und des strukturellen Aufbaus der vier Polymeren (Abb. 1) zusammengefaßt.

Polymer 1 wurde durch Addition der beiden D4R-Kieselsäurederivate $\left[\mathrm{H}\left(\mathrm{CH}_{3}\right)_{2} \mathrm{Si}\right]_{8} \mathrm{Si}_{8} \mathrm{O}_{20}\left(\mathrm{Q}_{8} \mathrm{M}_{8}{ }^{\mathrm{H}}\right)$ und $\left[\left(\mathrm{CH}_{2}=\mathrm{CH}\right)\left(\mathrm{CH}_{3}\right)_{2} \mathrm{Si}_{8} \mathrm{Si}_{8} \mathrm{O}_{20}\left(\mathrm{Q}_{8} \mathrm{M}_{8}{ }^{\mathrm{V}}\right)\right.$ erhalten. Der Umsatzgrad des Polymers liegt-bezogen auf die 8 Reaktionsstellen der D4R-Einheiten - bei 6.3. Verglichen mit den anderen Polymeren liegen in 1 die kürzesten kettenförmigen Brücken zwischen den D4R-Einheiten vor.

Polymer 2 wurde durch Addition von $\mathrm{Q}_{8} \mathrm{M}_{8}{ }^{\mathrm{H}}$ an Divinyltetramethyldisiloxan $\left(\mathrm{CH}_{2}=\mathrm{CH}\right)\left(\mathrm{CH}_{3}\right)_{2} \mathrm{Si}-\mathrm{O}-\mathrm{Si}\left(\mathrm{CH}_{3}\right)_{2}\left(\mathrm{CH}=\mathrm{CH}_{2}\right)$ mit dem sehr hohen Umsatzgrad von 7.8 hergestellt.

Polymer 3 wurde durch Addition des Tetramethylcyclotetrasiloxans $\left[\mathrm{HCH}_{3} \mathrm{SiO}_{4}\right.$ (in $10 \%$ igem Überschuß) an $\mathrm{Q}_{8} \mathrm{M}_{8}{ }^{\mathrm{V}}$ hergestellt. Der Umsatzgrad beträgt 7.4, bezogen auf die Komponente $\mathrm{Q}_{8} \mathrm{M}_{8}{ }^{\mathrm{V}}$.
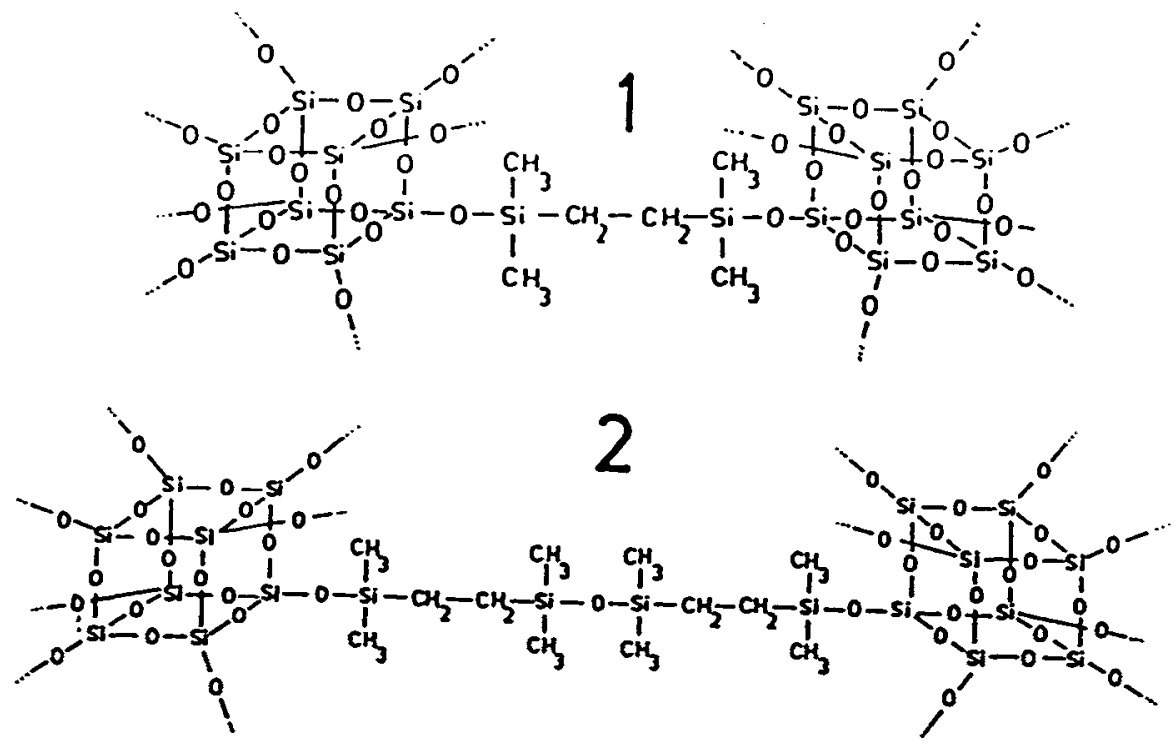

Abb. 1a Konstitutionsschema der Organokieselsäurepolymere 1, 2 

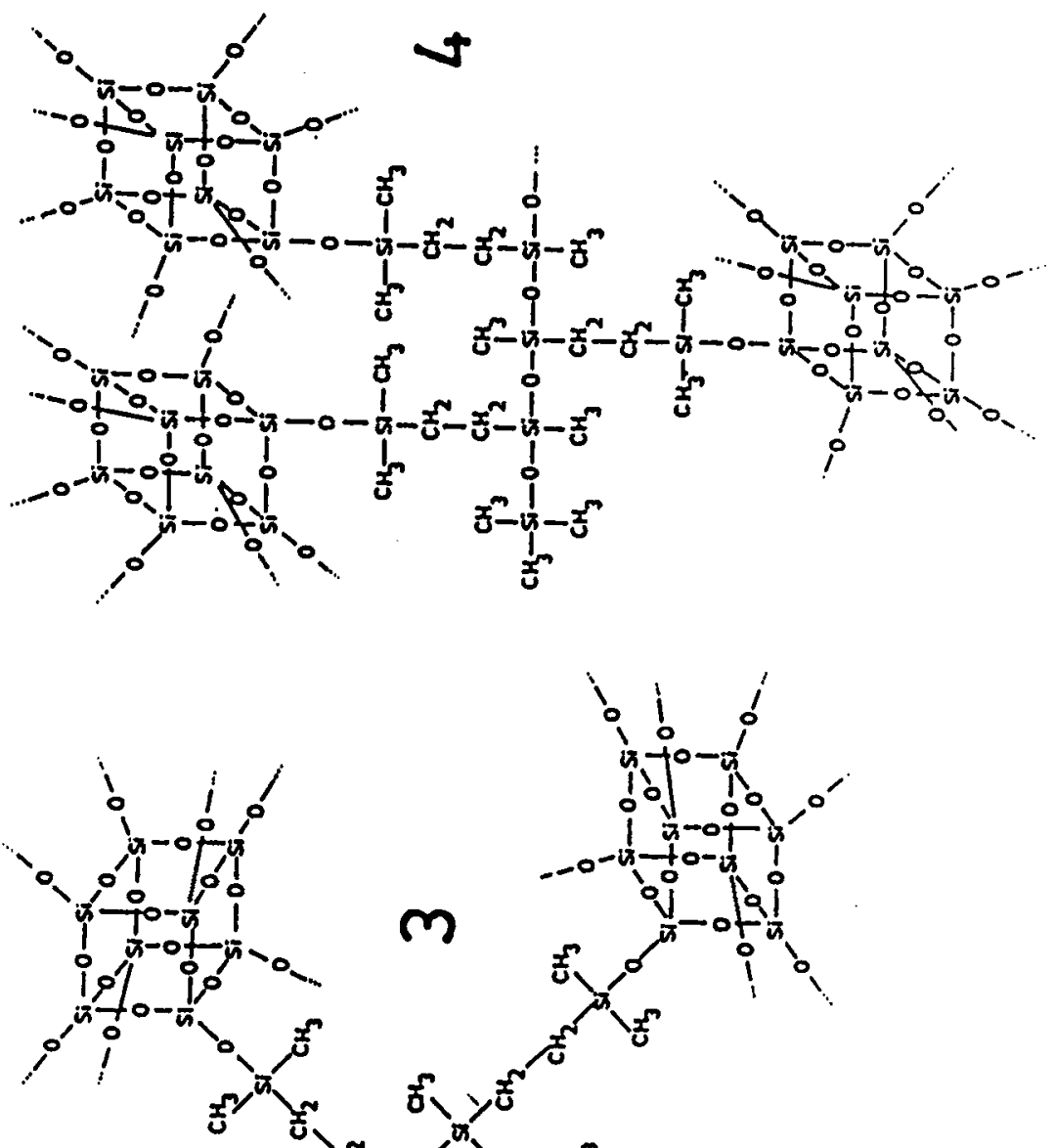

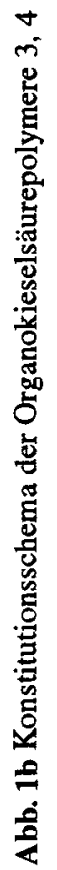

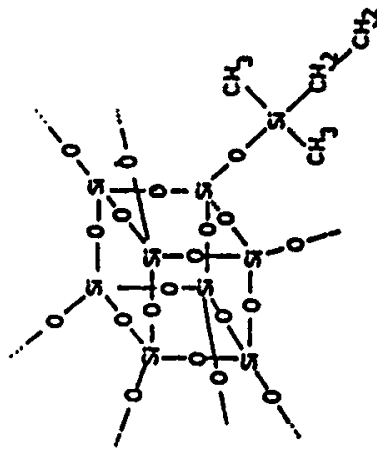


Polymer 4 wurde durch Addition eines Polymethylhydrogensiloxans mit einer mittleren Kettenlänge von $25\left(\mathrm{HCH}_{3} \mathrm{Si}-\mathrm{O}\right)$-Einheiten an $\mathrm{Q}_{8} \mathrm{M}_{8}{ }^{\mathrm{V}}$ erhalten. Polymer 4 weist mit 5.5 den niedrigsten Umsatzgrad auf.

Genauere Untersuchungen [1] ergaben, daß der Umsatzgrad kleiner als 8 hauptsächlich auf sterische Hinderungen bei den Additionsreaktionen zurückzuführen ist. Auf Grund der unvollständigen Reaktion liegen in den Polymeren noch unumgesetzte $(\mathrm{Si}-\mathrm{H})-$ bzw. $\left(\mathrm{CH}_{2}=\mathrm{CH}-\mathrm{Si}\right)$ - Gruppen vor, bei den Polymeren 1, 2 und 4 in äquivalenten Mengen, bei 3 mit einem Überschuß an (Si-H).

Die thermoanalytischen Untersuchungen erfolgten mittels simultaner TGDTA in Platintiegeln (Thermowaage SETARAM TAG 24) und Wärmeflußkalorimetrie in Aluminiumtiegeln (SETARAM DSC 92), jeweils mit Einwaagen von ca. $10 \mathrm{mg}$ im Luftstrom. Um meßtechnische Einflüsse (Auftrieb, Drift der Basislinie) zu korrigieren, wurden die Proben nach erstmaligem Aufheizen in einem zweiten Lauf unter den gleichen Bedingungen erneut erhitzt, die Meßkurven dieses zweiten Laufes wurden als Blindwert von denen des ersten Laufs subtrahiert. Bei den im folgenden dargestellten und diskutierten Ergebnissen handelt es sich hauptsächlich um Differenzkurven.

\section{Ergebnisse}

Thermoanalytische Messungen mit einer Aufheizrate von $1.5 \mathrm{deg} \cdot \mathrm{min}^{-1}$ lieferten für die vier Organokieselsäurepolymere die Kurven der Abb. 2-4. Die thermogravimetrischen Ergebnisse (Abb. 2) werden bei differentieller Auftragung (Abb. 3) besser vergleichbar mit denen der DTA (Abb. 4) oder DSC, zu denen sie annähernd spiegelbildlichen Verlauf aufweisen. Ausgangs-, Zwischen- und Endprodukte waren stets weiß gefärbt. Der exotherme Ablauf und die fast durchgängige Masseabnahme sprechen für das Vorliegen eines thermooxidativen Abbaus mit vollständiger Verbrennung der organischen Anteile, wobei eine Trennung in zwei Stufen im Bereich 200 bis $350^{\circ} \mathrm{C}$ bzw. 350 bis $600^{\circ} \mathrm{C}$ offensichtlich ist.

Abbildung 5 zeigt DSC-Kurven des Polymers 1 für unterschiedliche Aufheizraten. Um die Kurven besser vergleichen zu können, wurde die Meßempfindlichkeit mit abnehmender Aufheizrate um den angegebenen Faktor vergrößert. Während bei $10 \mathrm{deg} \cdot \mathrm{min}^{-1}$ nur eine unvollkommene, bei $1.5 \mathrm{deg} \cdot \mathrm{min}^{-1}$ eine deutliche Trennung in 2 große Teilschritte erkennbar ist, deutet sich bei 0.3 und $0.06 \mathrm{deg} \cdot \mathrm{min}^{-1}$ eine weitere Aufspaltung des ersten Teilschritts in zwei Teileffekte, des zweiten Teischritts in eine Vielzahl kleiner Sprünge an. Ferner erkennt man mit abnehmender Aufheizrate die allgemein von der Zersetzung vieler Polymerer bekannte Verschiebung der thermischen 


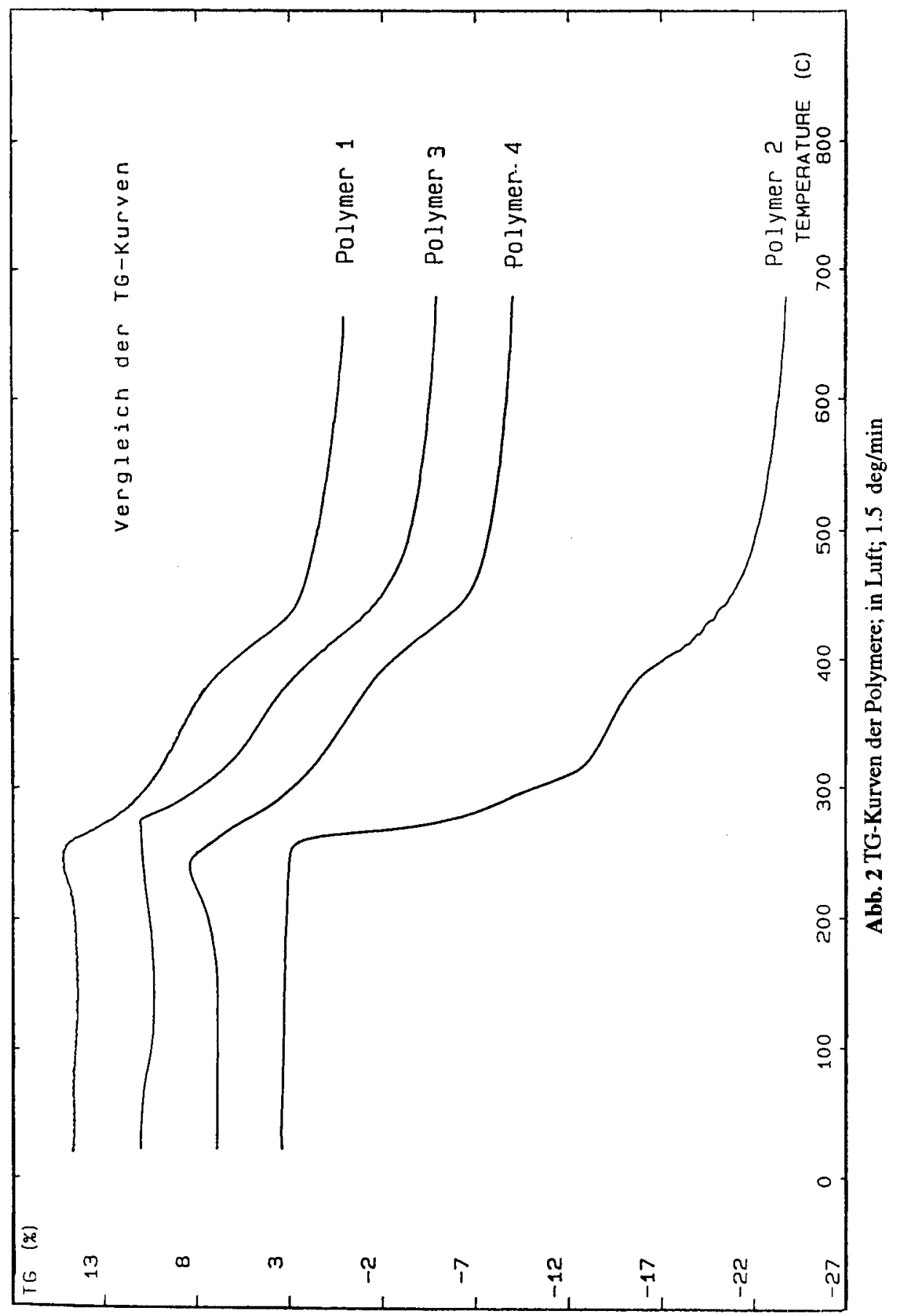




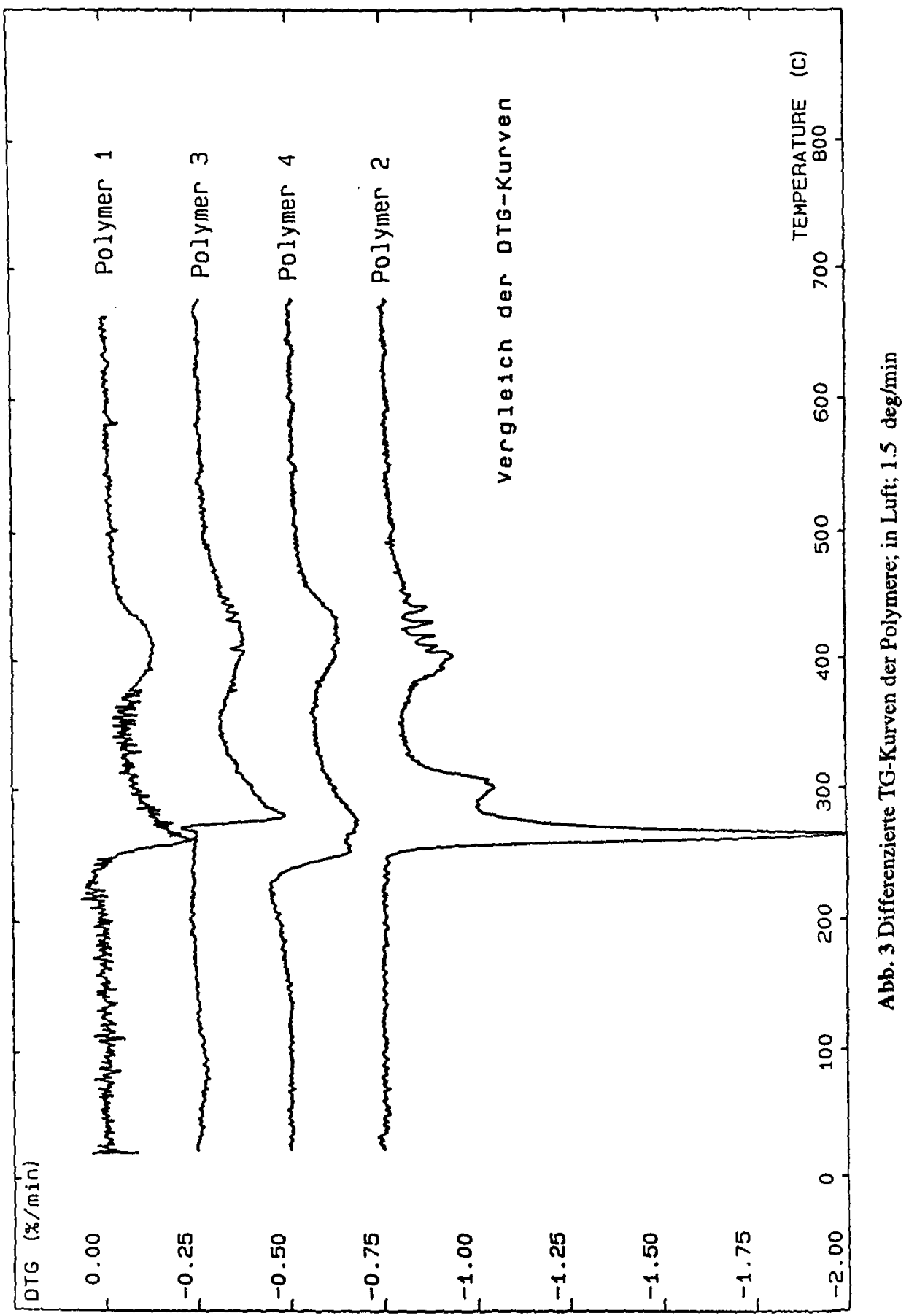




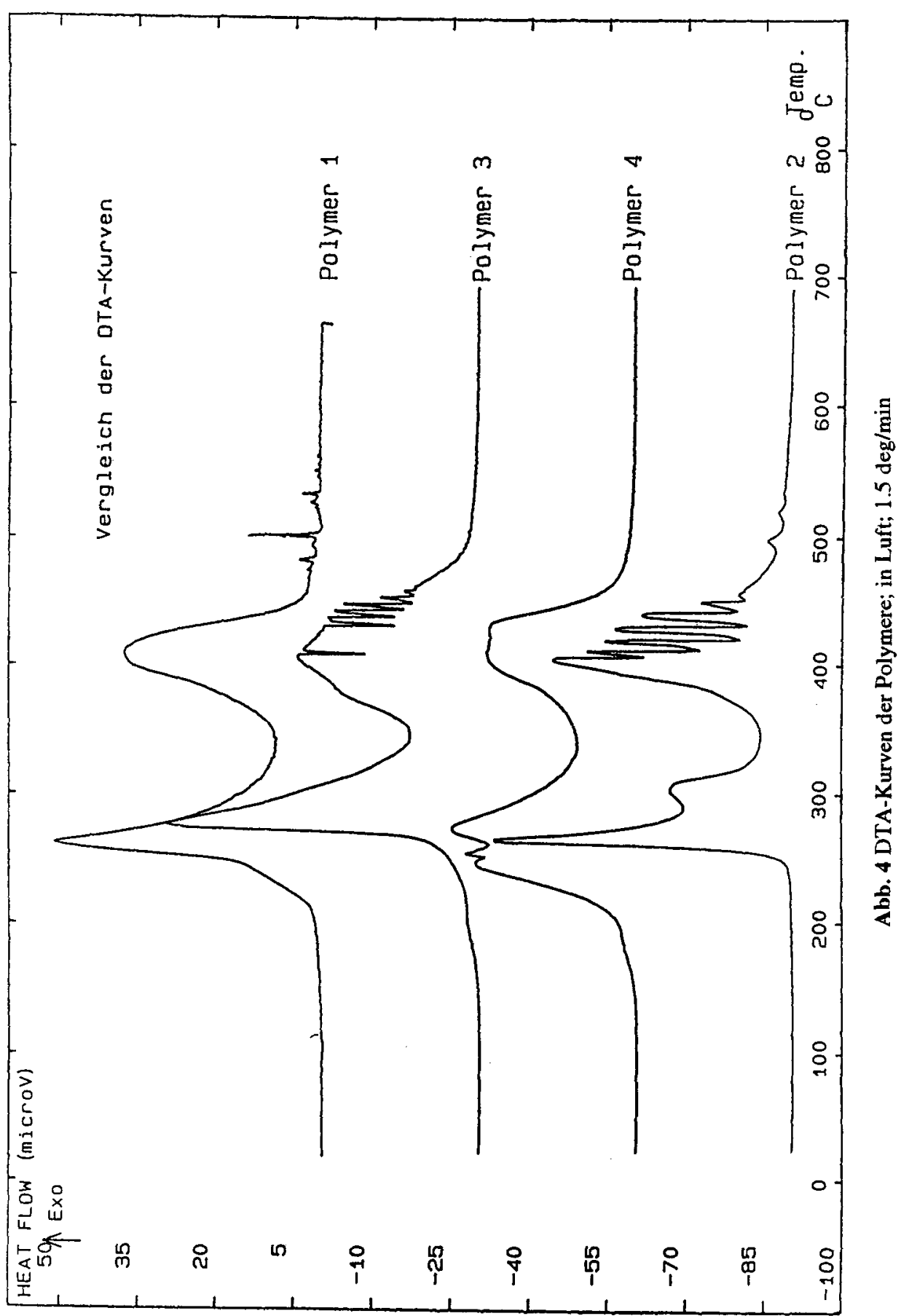


Effekte zu niedrigeren Temperaturen. Um mit einem erträglichen Aufwand an Meßzeit aussagefähige Ergebnisse zu erhalten, wurde die Mehrzahl der Messungen bei $1.5 \mathrm{deg} \cdot \mathrm{min}^{-1}$ durchgeführt.

Abbildung 6 zeigt einen Vergleich der Meßergebnisse von DSC- und TGDTA-Messungen am Polymer 1. Trotz der unterschiedlichen Meßgeräte und Tiegelmaterialien und deutlich abweichender Probengeometrien (TG-DTA: Schichtdicke ca. $4 \mathrm{~mm}$; DSC: $<1 \mathrm{~mm}$ ) wird ein weitgehend übereinstimmender Kurvenlauf erhalten, der eine Korrelation der Ergebnisse beider Methoden rechtfertigt.

\section{Diskussion}

Bei Betrachtung der Meßergebnisse in Abb. 2 findet man bei den Polymeren 1,3 und 4 oberhalb $200^{\circ} \mathrm{C}$ anfänglich eine geringe exotherme Massezunahme (bis $1.5 \%$ bei Polymer 4), die dann in eine ebenfalls exotherme starke Masseabnahme übergeht. Das zeigt, daß zunächst Sauerstoff addiert wird, bevor bei weiterer Temperaturerhöhung niedermolekulare Oxidationsprodukte $\left(\mathrm{CO}_{2}\right.$ und $\mathrm{H}_{2} \mathrm{O}$ ) eliminiert werden. Die Massezunahme $\delta m$ bis zum Maximum zeigt eine deutliche Korrelation mit dem Gehalt nicht umgesetzter ( $\mathrm{Si}-\mathrm{H}$ )-Gruppen in den Polymeren an (Tab. 1); $\delta m$ liegt aber deutlich unter dem für vollständige Umsetzung gemäß: $2(\mathrm{Si}-\mathrm{H})+\mathrm{O}_{2} \rightarrow 2(\mathrm{Si}-\mathrm{OH})$ zu erwartenden Wert. Vor der vollständigen Oxidation der ( $\mathrm{Si}-\mathrm{H})$-Gruppen setzt wahrscheinlich der thermooxidative Abbau ein, der mit Masseabnahme verbunden ist. In Polymer $2 \mathrm{mit}$ einem sehr geringen Gehalt an ( $\mathrm{Si}-\mathrm{H})$-Gruppen ist keine intermediäre Massezunahme zu beobachten, der exotherme Effekt setzt erst bei höherer Temperatur ein als bei den anderen Polymeren.

Tabelle 1 Massezunahme durch Oxidation der $\mathrm{Si}-\mathrm{H}-\mathrm{Bindungen}(\mathrm{Si}-\mathrm{H} \rightarrow \mathrm{Si}-\mathrm{OH}$ )

\begin{tabular}{ccc}
\hline Polymer & $\begin{array}{c}\text { Massezunahme } \\
\text { bis zum Maximum } \\
\delta m / \text { Masse-\% }\end{array}$ & $\begin{array}{c}\text { berechnete } \\
\text { Massezunahme } \\
\delta m / \text { Masse-\% }\end{array}$ \\
\hline 1 & 0.86 & 1.20 \\
2 & 0 & 0.18 \\
3 & 0.79 & 1.27 \\
4 & 1.52 & 2.27 \\
\hline
\end{tabular}




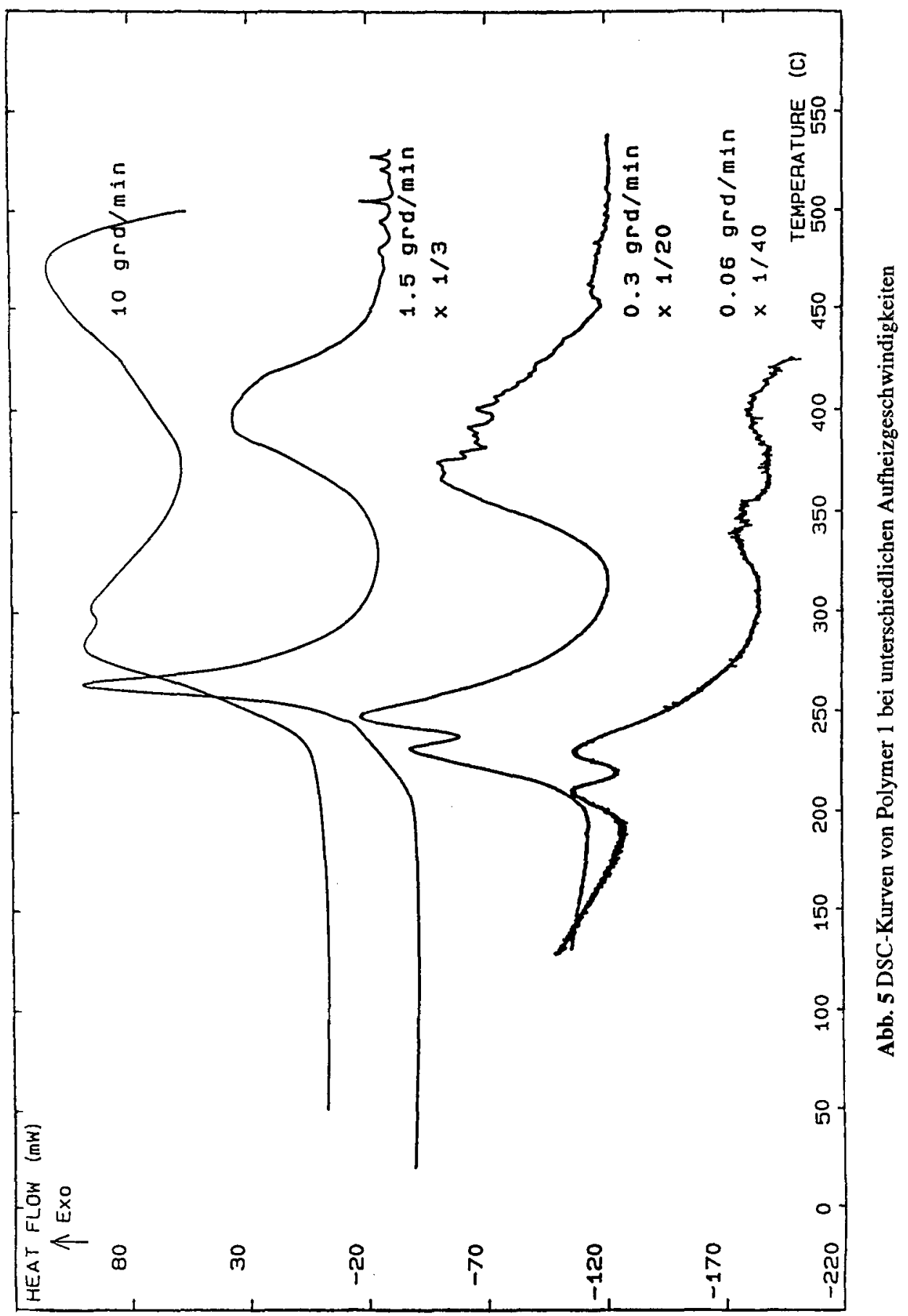




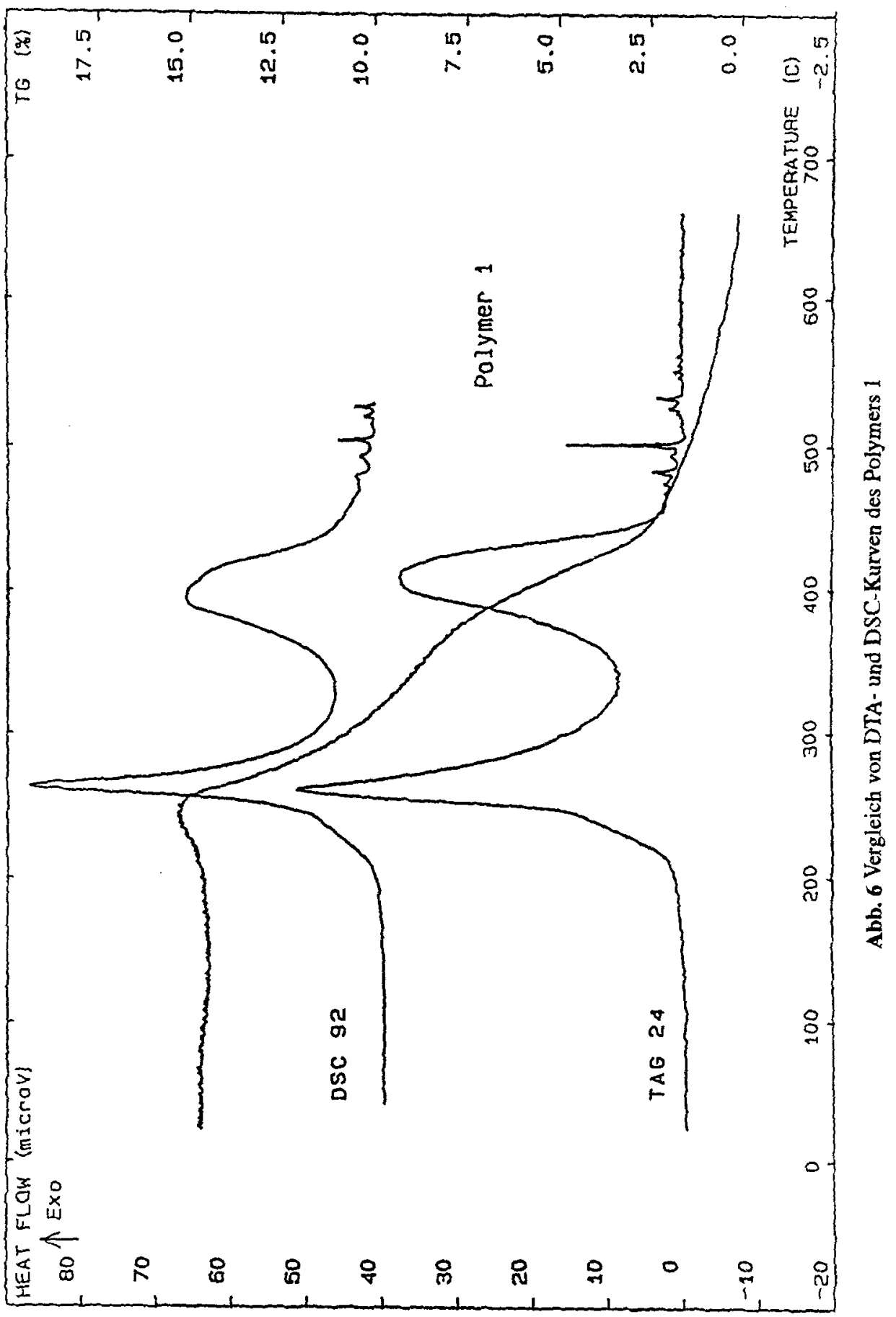


Für alle vier Polymere ist der thermooxidative Abbau in zwei Teilbereichen unterhalb bzw. oberhalb ca. $350^{\circ} \mathrm{C}$ charakteristisch. Bei ausreichend langsamer Aufheizrate unterscheiden sich diese Teilbereiche immer deutlicher. Es kann auf einen zweistufigen Abbaumechanismus geschlossen werden. Hinweise auf die Natur der Hauptreaktionen in den beiden großen Teilschritten des thermooxidativen Abbaus ergeben sich aus der ${ }^{29} \mathrm{Si}$-NMR-spektroskopischen Untersuchung von Zwischenprodukten, die durch Abschrecken von 1 nach Erhitzen auf 342 bzw. $450^{\circ} \mathrm{C}$ erhalten wurden, aus BET-Untersuchungen, aus Raman-Spektren sowie durch Vergleich von theoretisch berechneten Masseabnahmen und Verbrennungs-/Zersetzungsenthalpien mit den experimentellen Daten.

In Abbildung 7 sind die ${ }^{29} \mathrm{Si}$-NMR-Spektren des Ausgangspolymeren 1 und seiner Umsetzungsprodukte zusammengestellt. Abbildung 8 zeigt die mittels ${ }^{29} \mathrm{Si}-\mathrm{NMR}$ unterscheidbaren Struktureinheiten und ihre Kennzeichnung. Im Spektrum des thermisch unbehandelten Ausgangsprodukts findet man neben dem $Q^{4}$-Signal der D4R-Kieselsäureeinheiten $(\delta=-109.3 \mathrm{ppm})$ zwei Signale im Resonanzbereich für M-Si-Atome, die nicht umgesetzten Vinyl-Si- bzw. SiH-Gruppen ( $\delta=0$ bis $-2 \mathrm{ppm}$ ) sowie den Brücken-Si-Atomen zwischen den D4R-Einheiten $(\delta=13.1 \mathrm{ppm})$ zuzuordnen sind.

In der auf $342^{\circ} \mathrm{C}$ aufgeheizten Probe findet man nur noch wenig M-SiAtome neben viel neugebildeten $\mathrm{D}-(\delta \approx-20 \mathrm{ppm})$ und T-Si-Atomen $(\delta \approx-60 \mathrm{ppm})$. Das spricht dafür, daß im Verlaufe des ersten Teilschritts nach den besonders reaktiven ( $\mathrm{Si}-\mathrm{H}$ )-Gruppen vorrangig die nach ihrer Bindungsenergie relativ schwachen ( $\mathrm{Si}-\mathrm{CH}_{2}-\mathrm{CH}_{2}-\mathrm{Si}$ )-Brücken und vorhandenen VinylSi-Gruppen oxidieren und kondensieren. Die Anteile von T-Baugruppen werden auf die beginnende Weiteroxidation der $\left(\mathrm{O}-\mathrm{Si}\left(\mathrm{CH}_{3}\right)_{2}-\mathrm{O}\right)$-Gruppen zurückgeführt. Das ${ }^{29} \mathrm{Si}-\mathrm{NMR}-\mathrm{Spektrum}$ der bis $450^{\circ} \mathrm{C}$ erhitzten Probe weist nur noch ein kleines Signal im Resonanzbereich von T-Si-Atomen auf, das möglicherweise noch durch die Rotationsseitenbänder des dominierenden Signals der Q-Si-Atome überlagert wird. Das Q-Signal ist nicht mehr symmetrisch, sondern zeigt zwei Schultern, die entsprechend einer Kurvenzerlegung den Signalen von $\mathrm{Q}^{2}-$ (ca. $8 \%$ ) und $\mathrm{Q}^{3}$-Si-Atomen (ca. 26\%) zuzuordnen sind. Das intensitätsstärkste $\mathrm{Q}^{4}$-Signal (ca. 66\%) wird wahrscheinlich wieder durch die Si-Atome des D4R-Kieselsäuregerüstes sowie durch die bei der thermischen Oxidation neu gebildeten $\mathrm{Q}^{4}-\mathrm{Si}$-Atome hervorgerufen. $\mathrm{Ab}$ gesehen von dem kleinen T-Signal sind nach der $450^{\circ} \mathrm{C}$ Behandlung in dem Polymer 1 praktisch keine $\mathrm{Si}-\mathrm{C}$-Bindungen mehr nachzuweisen.

Für das Polymer 1 wurde eine wesentlich größere Oberfläche vergleichsweise zu den Polymeren 2-4 festgestellt [4]. Diese wird auf die Ausbildung von Raumnetzstrukturen in Gegenwart von kurzen (Ethylen)-brücken zwischen den 
D4R-Einheiten zurückgeführt. Nach der thermischen Behandlung des Polymers 1 ändern sich die Oberflächen, wie in Tab. 2 angegeben. Die Ergebnisse zeigen eine mit steigender Temperatur kleiner werdende Oberfläche an, wobei nach oxidativem Abbau der Ethylengruppen bei $342^{\circ} \mathrm{C}$ eine noch bemerkenswert große Oberfläche existiert, die durch die eng verknüpften D4R-Kieselsäureeinheiten wahrscheinlich noch erhalten ist. Mit der weiteren Temperaturerhöhung auf $450 \mathrm{bzw} .900^{\circ} \mathrm{C}$ schließlich, nach dem oxidativem $\mathrm{Ab}$ bau der Methylgruppen, erfolgt Gitterzusammenbruch, so daß diese Produkte nur eine kleine Oberfläche aufweisen.

Tabelle 2 Oberflächenbestimmung für Polymer 1 nach BET $\left(\mathbf{N}_{2}\right)$

\begin{tabular}{|c|c|c|c|c|}
\hline \multirow{2}{*}{$\begin{array}{l}\text { Thermisch } \\
\text { Temperatur } /^{\circ} \mathrm{C}\end{array}$} & \multirow{2}{*}{$\frac{\text { Unbehandelt }}{-}$} & \multicolumn{3}{|c|}{ Behandelt } \\
\hline & & 342 & 450 & 900 \\
\hline Oberfläche $/ \mathrm{m}^{2} \cdot \mathrm{g}^{-1}$ & 190 & 112 & 7.9 & 5.0 \\
\hline
\end{tabular}

An den gleichen Proben, von denen die Oberflächen ermittelt wurden, erfolgte die Aufnahme von Ramanspektren. Dabei konnte festgestellt werden:

- Für das thermisch unbehandelte Polymer 1 wurden Banden der $\mathrm{C}-\mathrm{H}$ Schwingungen der $\mathrm{Si}-\mathrm{C}$ - sowie der nicht umgesetzten $(\mathrm{Si}-\mathrm{H})$ - und ( $\mathrm{Si}-$ $\mathrm{CH}=\mathrm{CH}_{2}$ )-Gruppen registriert. Der Methyl- und Ethylen-Anteil in den $\mathrm{Si}-\mathrm{C}$-Schwingungen läßt sich allerdings nicht differenzieren.

- Das bei $342^{\circ} \mathrm{C}$ behandelte Produkt weist $\mathrm{C}-\mathrm{H}$-Schwingungen in reduzierten Maße auf. Si-C-Schwingungen sind nicht mehr nachweisbar und evtl. im Untergrund verdeckt. Die Abnahme an $\mathrm{C}-\mathrm{H}$-Schwingungen deutet wohl auf Verluste hin, nicht spezifisch jedoch auf den Verlust der Ethylenbrücken.

- Im Spektrum des bei $450^{\circ} \mathrm{C}$ behandelten Polymers sind keinerlei Hinweise auf organische Anteile mehr enthalten.

- Nach der Behandlung bei $900^{\circ} \mathrm{C}$ weist das Produkt ein ähnliches Spektrum wie $\mathrm{SiO}_{2}$-Gläser auf. Die Zwischenstufen des oxidativen Abbaus der organischen Anteile sind mittels Raman-Spektroskopie nicht zu unterscheiden.

Die Deutung, wonach der erste Teilschritt hauptsächlich der Oxidation der ( $\mathrm{Si}-\mathrm{CH}_{2}-\mathrm{CH}_{2}-\mathrm{Si}$ )-Brücken, der zweite der $\left(\mathrm{Si}-\mathrm{CH}_{3}\right)$-Gruppen zuzuordnen ist, wird durch einen Vergleich experimentell ermittelter und berechneter Masseänderungen und Verbrennungs-/Zersetzungsenthalpien bestätigt (Tab. 3). Eine Abweichung davon liegt bei Polymer 2 vor. Hier erkennt man, daß der erste Schritt des Abbaus deutlich in zwei Teile aufgespalten ist. Dem berechneten Masseverlust für den ersten Teilschritt der Oxidation der $\left(\mathrm{Si}-\mathrm{CH}_{2}-\mathrm{CH}_{2}-\mathrm{Si}\right)$ Brücken (5.5\%) steht der experimentell gemessene Wert von $11.64 \%$ 
KÖLSCH et al.: ORGANOKIESELSÄUREPOLYMEREN

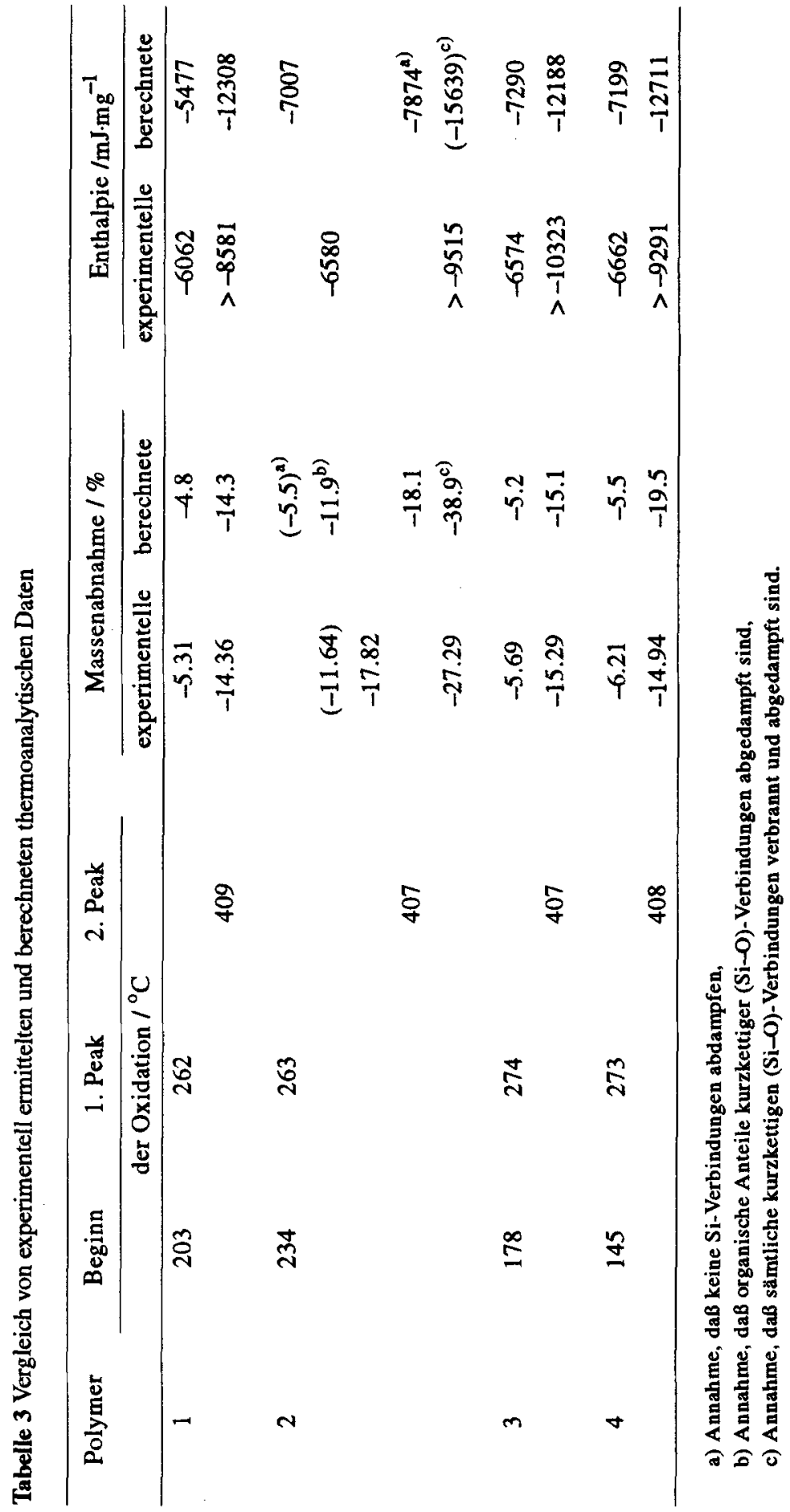




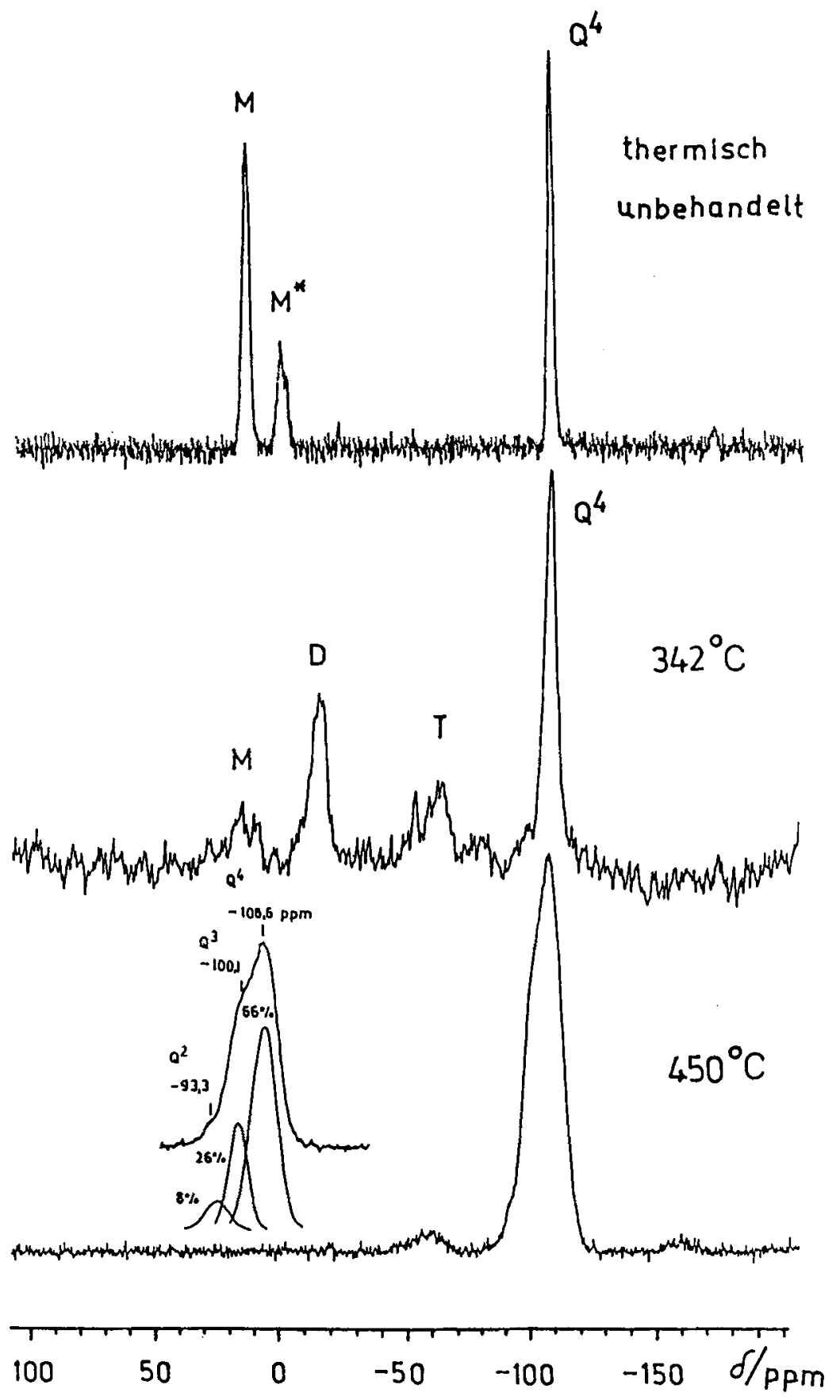

Abb. $7{ }^{29} \mathrm{Si}$-NMR-Spektren von thermisch behandeltem Polymer 1 


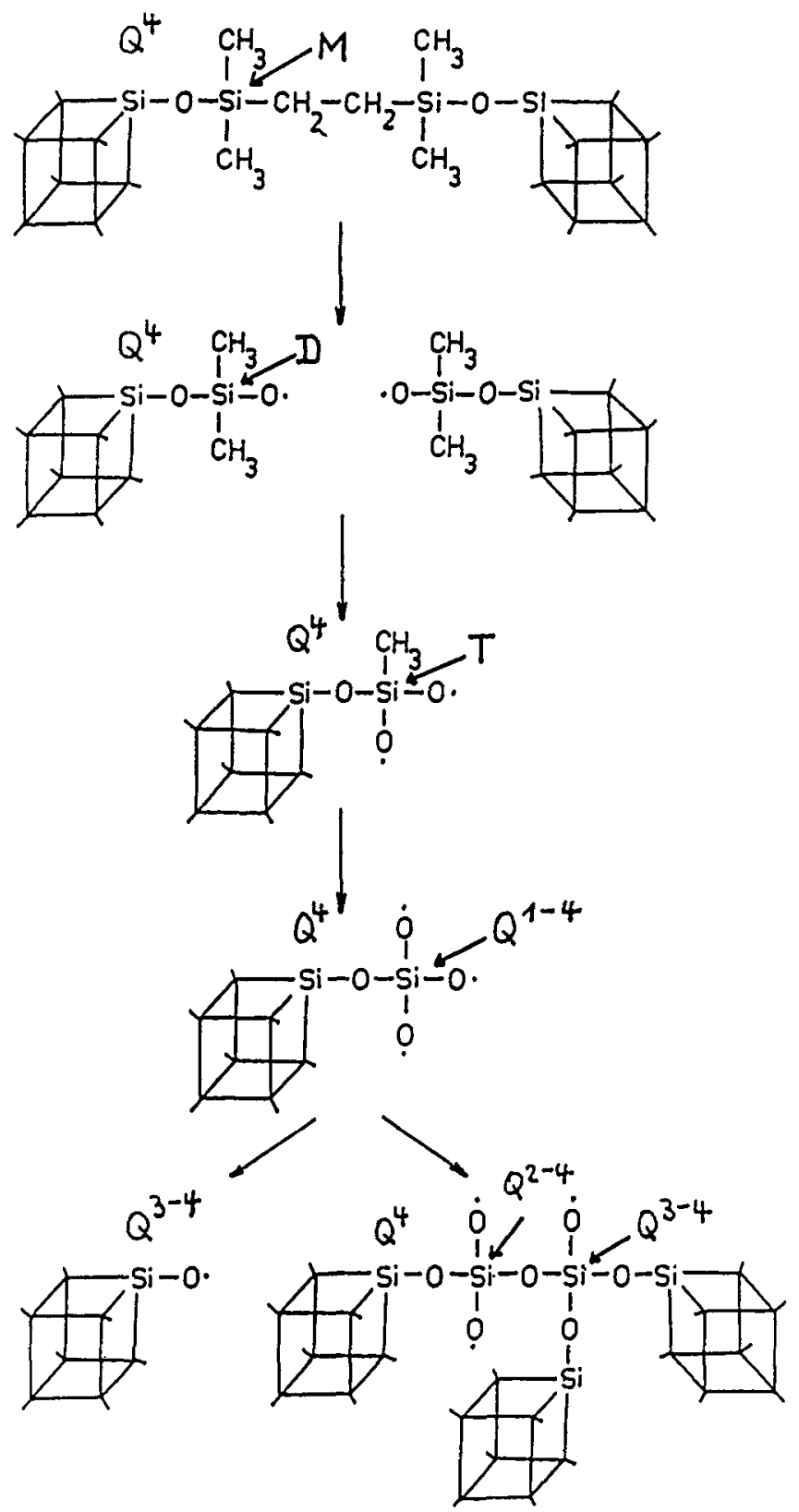

Abb. 8 Schema der Bildung von $\mathrm{Si}-\mathrm{O}-\mathrm{Struktureinheiten} \mathrm{bei} \mathrm{der} \mathrm{thermischen} \mathrm{Behandlung}$ des Polymers 1 
gegenüber. Es wird angenommen, daß kurzkettige flüchtige Si-haltige Produkte den höheren Gewichtsverlust verursachen. Nur Polymer 2 enthält Baugruppen der Struktur $-\mathrm{CH}_{2}-\mathrm{CH}_{2}-\mathrm{Si}\left(\mathrm{CH}_{3}\right)_{2}-\mathrm{O}-\mathrm{Si}\left(\mathrm{CH}_{3}\right)_{2}-\mathrm{CH}_{2}-\mathrm{CH}_{2}-$, bei deren Zersetzung vermutlich flüchtige Siloxan-Produkte entstehen. Bezieht man deren Entweichen in die Bilanz ein, so wird ein Gewichtsverlust von $11.9 \%$ erhalten und dieser Wert wiederum stimmt mit dem berechneten nahezu überein.

In der 2. Stufe verbrennen hauptsächlich die Methylgruppen der Dimethylsiloxan- $\left[\mathrm{Si}\left(\mathrm{CH}_{3}\right)_{2}-\mathrm{O}\right]$-Baueinheiten. Auch hier wird gute Übereinstimmung von experimentellen und berechneten Werten gefunden. Abweichend verhält sich wiederum Polymer 2.

Der gesamte Masseverlust nach Verbrennung der Methylgruppen des Polymers 2 ist mit $27.29 \%$ deutlich höher als bei vollständiger Verbrennung $\mathrm{zu} \mathrm{SiO}_{2}$ zu erwarten wäre (18.10\%). Unter der Annahme, daß die gesamten Baugruppen $\mathrm{CH}_{2}-\mathrm{CH}_{2}-\mathrm{Si}\left(\mathrm{CH}_{3}\right)_{2}-\mathrm{O}-\mathrm{Si}\left(\mathrm{CH}_{3}\right)_{2}-\mathrm{CH}_{2}-\mathrm{CH}_{2}$ verdampfen, ist ein Gewichtsverlust von $38.9 \%$ zu erwarten. Der experimentell ermittelte Wert liegt zwischen diesen Werten. Teile der kleinen Baugruppen sind wahrscheinlich verdampft, ein anderer Teil der $\mathrm{Si}-\mathrm{O}$-Gruppen kondensiert.

Ein ähnliche Parallelität wie bei den gravimetrischen Ergebnissen wird auch für die experimentell ermittelten und berechneten Verbrennungsenthalpien gefunden. Durch Addition der in der Tab. 4 aufgeführten Inkrement-Verbrennungs- und Spaltungsenthalpien wurden die Gesamt-Verbrennungswärmen der Polymeren errechnet. Diese Werte sind in der Tab. 3 den experimentell ermittelten gegenübergestellt. Bei der einfachen Summation der Wärmeinkremente, die näherungsweise ermittelt wurden, sind systematische und methodische Fehler enthalten. Die berechneten Verbrennungsenthalpien sind bis auf eine Ausnahme (Polymer 1, 1. Stufe) größer als die experimentell ermittelten. Auf die Ausnahme wird unten eingegangen. Die zu großen Werte sind neben der bereits genannten Fehlerquelle hauptsächlich auf kalorisch nicht erfaßte Verbrennungswärmen im DSC sowie dessen Temperaturbegrenzung bis $550^{\circ} \mathrm{C}$ zurückzuführen. Die 2. Stufe der Verbrennung ist bei dieser Temperatur noch nicht abgeschlossen, die experimentell ermittelten Verbrennungswärmen müssen daher zu klein ausfallen. Trotz der genannten Fehlerquellen kann man eine befriedigende Übereinstimmung von experimentell ermittelten und berechneten Verbrennungswärmen feststellen.

Infolge der partiellen Verdampfung Si-haltiger Produkte aus dem Polymeren 2 liefern diese keinen Beitrag zur Verbrennungswärme, vielmehr wird durch das Verdampfen noch Wärme verbraucht. Für die an D4R-Kieselsäureeinheiten noch enthaltenen Methylgruppen des Polymers 2 berechnet sich eine Verbrennungswärme von $7874 \mathrm{~mJ} / \mathrm{mg}$. Wären keine Si-organischen Produkte verdampft, müßte eine Wärme von $15639 \mathrm{~mJ} / \mathrm{mg}$ gefunden werden. Der experimentell gefundene Wert liegt dazwischen. Das unterstützt die Annahme, daß 
einerseits siliciumorganische Zersetzungsprodukte verdampft sind und anderseits ein Teil, zu $\mathrm{SiO}_{2}$ oxidiert, erhalten bleibt. Die gravimetrischen Ergebnisse gehen mit dieser Annahme konform.

Tabelle 4 Inkrementverbrennungs- bzw. Spaltungsenthalpien ${ }^{\text {a) }}$

\begin{tabular}{lcccccc}
\hline $\begin{array}{l}\text { Inkrement } \\
\text { Verbrennungs- }\end{array}$ & $(-\mathrm{H})$ & $\left(-\mathrm{CH}_{3}\right)$ & $\left(-\mathrm{C}_{2} \mathrm{H}_{3}\right)$ & $\left(-\mathrm{CH}_{2}-\right)$ & $(-\mathrm{O}-\mathrm{Si} \equiv)$ & $(\equiv \mathrm{Si}-\mathrm{C} \equiv)$ \\
$\begin{array}{l}\text { Bidungsenthalpie / } \\
\text { kJ-mol }\end{array}$ & -121.0 & -780.8 & -1290.0 & -705.5 & -108.2 & +28.0 \\
\hline
\end{tabular}

a)Aus Verbrennungs- bzw. Bildungswärmen von $\mathrm{H}_{2}, \mathrm{CH}_{4}, \mathrm{C}_{2} \mathrm{H}_{4}$ und $\mathrm{SiC}$ näherungsweise ermittelt

Im folgenden soll versucht werden, die bei langsamer Aufheizung besonders deutlich erkennbare Feinstruktur der beiden Teilschritte in den TG-, DSC- und DTA-Kurven zu deuten (Abb. 2-5). Der erste Verbrennungs-peak bei den untersuchten Polymeren spaltet sich in zwei Teile auf. Darin kommt die anfängliche Oxidation der $\mathrm{Si}-\mathrm{H}$ - und evtl. unumgesetzter Vinyl-Gruppen zum Ausdruck. Denkbar wäre auch eine schrittweise oxidative Spaltung der $\left(\mathrm{Si}-\mathrm{CH}_{2}-\mathrm{CH}_{2}-\mathrm{Si}\right)-$ Brücke an den beiden $\mathrm{Si}-\mathrm{C}$-Bindungen.

Die vielen kleinen Sprünge im zweiten peak, die sowohl gravimetrisch als auch kalorimetrisch nachweisbar sind, können eventuell auf eine schrittweise Verbrennung der einzelnen Methylgruppen zurückzuführen sein. Eine Zuordnung der Verbrennungs-peaks zur Gesamtzahl unterschiedlicher Methylgruppen der Polymeren ist allerdings nicht möglich, zumal bei verschiedenen Aufheizgeschwindigkeiten die Vorgänge sehr unterschiedlich ablaufen, wie es auch aus den Meßkurven der Abb. 5 zum Ausdruck kommt.

Eine alternative Deutung der Aufspaltung in schmale Sprünge setzt eine Art von oszillierender Reaktion voraus, bei der ein gasundurchlässiges Reaktionsprodukt an der Oberfläche zunächst die weitere Oxidation verhindert. Im Probeninnern geht jedoch die endotherme Zersetzung weiter, bis der steigende Druck der Zersetzungsprodukte die Deckschicht sprengt und erneut exotherme Oxidation stattfindet. Dabei entsteht wieder eine undurchlässige Oberflächenschicht und die Oxidation kommt abermals zum Stillstand usw. Eine sichere Interpretation der thermoanalytisch erkennbaren Teilschritte bedarf weiterer Untersuchungen, insbesondere an abgeschreckten Zwischenprodukten.

An weiteren Organokieselsäurepolymeren, die ebenfalls $\left(\mathrm{Si}-\mathrm{CH}_{2}-\mathrm{CH}_{2}-\mathrm{Si}\right)-$ Brücken neben anderen organischen Gruppen enthalten und die hier nicht behandelt wurden [7], wurden bei thermoanalytischen Messungen in Luft auch jeweils zwei Verbrennungsstufen ermittelt. Davon und von den Ergebnissen dieser Arbeit ausgehend wird verallgemeinert, daß in den ersten Verbren- 
nungsstufen stets die ( $\left.\mathrm{Si}-\mathrm{CH}_{2}-\mathrm{CH}_{2}-\mathrm{Si}\right)$-Gruppen, in den zweiten Stufen die Methylgruppen bzw. restlichen organischen Anteile oxidiert werden. Die qualitativen Analysen der gravimetrischen und DSC-Kurven (Kurvenverlauf, Beginn der Verbrennung usw.) können genutzt werden, um Aussagen zur Strukturzuordnung und der Reproduzierbarkeit von synthetisierten Organosilicaten zu machen. Die thermische Analyse bietet damit für diese Stoffklasse eine geeignete Charakterisierungsmethode.

Die Autoren danken den Herren Dr. W. Pilz und Dr. M. Noack für die Durchführung der Raman- und BET-Messungen sowie die Diskussion der Ergebnisse. Dem Fonds der chemischen Industrie wird für finanzielle Unterstützungen gedankt.

\section{Literatur}

1 D. Hoebbel, I. Pitsch, D. Heidemann, H. Jancke und W. Hiller, Z. Anorg. Allg. Chem., 583 (1990) 133.

2 P. A. Agaskar, J. Am. Chem. Soc., 111 (1989) 6858; J. Chem. Soc. Chem. Commun., (1992) 1024.

3 D. Hoebbel, I. Pitsch und D. Heidemann, Z. Anorg. Allg. Chem., 592 (1991) 207.

4 D. Schultze, P. Kölsch, M. Noack, P. Toussaint, D. Hoebbel und I. Pitsch, Z. Anorg. Allg. Chem., 612 (1992) 137.

5 D'Ans-Lax, Taschenbuch für Chemiker und Physiker, Springer Verlag, 1967.

6 Landolt-Börnstein: Zahlenwerte und Funktionen, 4. Teil, Kalorische Zustandsgrößen, Springer-Verlag 1961.

7 P. Kölsch, unveröffentlichte Ergebnisse.

Zusammenfassung - Durch Addition von Vinyl- und H-substituierten Doppelvierringkieselsäurederivaten bergestellte Organokieselsäurepolymere werden thermoanalytisch mit TG-, DTAand DSC-Messungen charakterisiert. Der thermooxidative Abbau der vier untersuchten Polymere erfolgt in mehreren Schritten: Oxidation der ( $\mathrm{Si}-\mathrm{H})$-Gruppen, Oxidation der Ethylenbriicken $\left(\mathrm{Si}-\mathrm{CH}_{2}-\mathrm{CH}_{2}-\mathrm{Si}\right)$ und Oxidation der $\left(\mathrm{Si}-\mathrm{CH}_{3}\right)$-Gruppen, jeweils unter Bildung von neuen ( $\mathrm{Si}-\mathrm{O}-\mathrm{Si}$ )-Bindungen. ${ }^{29} \mathrm{Si}-\mathrm{NMR}$-spektroskopische Ergebnisse stützen die Deutung des Reaktionsablaufsablaufes. Thermoanalytische Untersuchungsmethoden können erfolgreich zur Charakterisierung von Organokieselsäurepolymeren eingesetzt werden. 